\title{
Augmentation of Psychotherapy with Neurobiological Methods: Current State and Future Directions
}

\author{
Elisabeth Hertenstein ${ }^{a} \quad$ Ersilia Trinca $^{a} \quad$ Carlotta L. Schneider ${ }^{a}$ \\ Marina Wunderlin ${ }^{b}$ Kristoffer Fehér ${ }^{a}$ Dieter Riemann ${ }^{c}$ Christoph Nissen ${ }^{a}$ \\ aUniversity Hospital of Psychiatry and Psychotherapy, Bern, Switzerland; bUniversity Hospital of Old Age Psychiatry \\ and Psychotherapy, University of Bern, Bern, Switzerland; 'CClinic of Psychiatry and Psychotherapy, Medical Center - \\ University of Freiburg, Faculty of Medicine, University of Freiburg, Freiburg, Germany
}

\section{Keywords}

Neurobiology · Psychotherapy · Research Domain Criteria

\begin{abstract}
Background: Psychotherapy and pharmacotherapy are first-line treatments for mental disorders. Despite recent improvements, only approximately $50 \%$ of the patients reach sustained remission, indicating a need for novel developments. The main concept put forward in this systematic review and hypothesis article is the targeted coadministration of defined neurobiological interventions and specific psychotherapeutic techniques. Methods: We conducted a systematic literature search for randomized controlled trials comparing the efficacy of augmented psychotherapy to psychotherapy alone. Results: Thirtyfive trials fulfilled the inclusion criteria. The majority (29 trials) used augmentation strategies such as D-cycloserine, yohimbine, or sleep to enhance the effects of exposure therapy for anxiety disorders. Fewer studies investigated noninvasive brain stimulation with the aim of improving cognitive control, psychedelic compounds with the aim of enhancing existentially oriented psychotherapy, and oxytocin to improve social communication during psychotherapy. Results demonstrate small augmentation effects
\end{abstract}

for the enhancement of exposure therapy - however, some of the studies found negative results. Other methods are less thoroughly researched, and results are mixed. Conclusions: This approach provides an open matrix for further research and has the potential to systematically guide future studies.

(c) 2021 S. Karger AG, Basel

\section{Introduction}

Mental disorders are among the most prevalent and impairing disorders worldwide [1]. Current treatment guidelines include pharmacotherapy and psychotherapy as first-line treatments (e.g., [2]). Yet, despite some progress, less than half of the patients reach sustained remission with current standard treatments, indicating the need for novel developments [1].

This work aims at further promoting a systematic research concept for neurobiological augmentation of psychotherapy. We first summarize current developments in neurobiology and psychotherapy and illustrate that, despite several improvements, their interplay remains characterized by ideological and methodical distance. Still, major neurobiological developments do not directly
Correspondence to:

Elisabeth Hertenstein, elisabeth.hertenstein@upd.ch 
refer to psychotherapy, and today's standard psychotherapeutic treatment packages have mostly broad and insufficiently defined neurobiological mechanisms, limiting the potential for targeted augmentation. The main concept put forward in this work is the targeted coadministration of defined neurobiological interventions and specific psychotherapeutic techniques. An example is the augmentation of exposure and response prevention, the gold standard psychotherapeutic treatment for anxiety disorders, with the NMDA receptor agonist D-cycloserine that boosts fear extinction learning $[3,4]$. We aim at providing an agenda for future augmentation research and potential clinical implementation.

\section{Current Neurobiology Research}

A recent and widely recognized framework for mental health research is the Research Domain Criteria (RDoC) concept of the American National Institute of Mental Health (NIMH). Here, the RDoC framework is used as an example for the identification of core neurobiological domains of mental health. The aim of the $\mathrm{RDoC}$ is to identify basic domains of mental functioning and their underlying neurobiological mechanisms beyond diagnostic categories [5]. This is motivated by the experience that the descriptive approach of the current versions of the International Classification of Diseases (ICD-10) and the American Diagnostic and Statistical Manual (DSM-5) have contributed to clinically important and broadly accepted diagnostic categories but also to the experience that these categories comprise inhomogeneous constellations of mental functioning and map only poorly to neurobiological mechanisms. The $\mathrm{RDoC}$ identifies 6 core domains of mental functioning, that is, negative and positive valence systems, cognitive systems, social processes, arousal systems, and sensorimotor systems. These domains are described in a matrix at different levels of analysis, namely, genes, molecules, cells, circuits, physiology, behavior, self-report, and paradigms. Following a dimensional rather than a categorical approach, this conceptualization might enable better characterization of neural mechanisms and promote more personalized health care through individual characterization of functioning on the 6 domains. However, the RDoC has been developed from a neurobiology perspective and, as many other neuroscience developments, does not provide a direct link to psychotherapy.

\section{Current Psychotherapy Research}

Psychotherapy is subdivided into different schools, the most well-known being psychodynamic psychotherapy and (cognitive) behavioral therapy [6]. Other psychotherapeutic schools include systemic psychotherapy [7] and person-centered psychotherapy [8]. Newer psychotherapeutic approaches using concepts such as (self-)acceptance and mindfulness, for example, acceptance and commitment therapy [9] and mindfulness-based cognitive therapy [10], are allocated to the so-called "third wave of behavioral therapy." Characteristics of psychodynamic psychotherapy are the assumption that insights are critical for therapeutic success, the objective of bringing unconscious content into consciousness, the identification of assumed defense mechanisms, and a focus on early formative experiences. Characteristics of behavioral therapy are a background in learning theory: the identification of causes and consequences of behavior, the assumption that behavioral and cognitive changes are critical for therapeutic success, and a focus on present conditions, rather than early experiences. Whereas these 2 main schools have traditionally been perceived as divided and largely incompatible, more recent developments, such as schema therapy and the cognitive behavioral analysis system of psychotherapy, successfully combine aspects of both approaches.

Regardless of differences between the schools, it can be assumed that effective psychotherapy leads to some longer-term changes of inner experiences and interaction patterns with the environment and therefore emerges from functional and structural (i.e., neuroplastic) changes in the brain. Animal research supports the view that behavioral interventions such as fear exposure play a critical role in the reorganization of neural networks [11]. In support of this view, psychotherapy has measurable effects on the brain, which in part resemble effects of successful pharmacotherapy $[12,13]$. These effects include activity changes in key brain regions for different disorders, such as a decreased metabolism in the caudate nucleus after psychotherapy in patients with obsessivecompulsive disorder (OCD) and decreased amygdala activity after exposure therapy in patients with anxiety disorders [13]. Following these lines, mental disorders can be conceptualized as alterations of neuronal network functioning, and neuroplastic changes in the brain can be viewed as a final common pathway for treatment effects. This opens the opportunity to use neurobiological augmentation strategies for the improvement of psychotherapy. Yet, still, most psychotherapeutic interventions are 
developed and applied without particular consideration of underlying neural processes. The low attention to psychotherapy in the neurosciences and vice versa still limits progress toward a systematic neurobiological augmentation of psychotherapy.

\section{Prior Approaches to Integrate Neurobiology and Psychotherapy}

The idea of identifying neural correlates of mental processes and improving mental health through neurobiological interventions is an old one. Since at least the 19 th century, it became tentative to translate the emerging neurobiological insights to mental health. Sigmund Freud, for instance, started with an interest in the function and structure of the brain. Yet, the neurobiological knowledge at the time was largely insufficient to measure or modulate individual psychological experiences. Freud later shifted to the development of psychoanalysis [14], which, as well as behavior therapy [15] and later cognitive behavioral therapy, evolved largely without direct reference to the neurosciences. In turn, parallel advances in biological psychiatry, such as in psychopharmacology, initially did not relate to psychotherapy. Moreover, some poorly refined concepts in the field of psychotherapy and some biological interventions that have legitimately been abandoned today, such as lobotomy (i.e., the neurosurgical dissection of connections from and to the frontal cortex for which Antonio E. Moniz received the Nobel Prize for Physiology and Medicine in 1949), increased the distance between the fields.

The Austrian psychologist Hubert Rohracher, who was critical of psychoanalysis as well as behaviorism, was one of the first to posit that the understanding of neurophysiology would represent a prerequisite for a successful scientific investigation of mental processes [16]. Niels Birbaumer, a former trainee of Rohracher, extended this work, for example, with the description of neural correlates of empathy and neurofeedback training $[17,18]$. The psychotherapy researcher Klaus Grawe introduced the term "neuropsychotherapy" [19]. He presented a new form of psychotherapy across different schools, based on the assumption that psychotherapy evokes the formation of novel and more adaptive memories and elicits neuroplastic changes in the brain. The neuroscientist Eric Kandel, who received the Nobel prize for his work on basic mechanisms of synaptic plasticity in the nautilus Aplysia californica, had a great interest in psychoanalysis as a student.
He later combined these fields and required a paradigm shift, demanding that psychotherapy must be underpinned by a thorough understanding of its neurobiological mechanisms [20,21].

Recently, psychotherapy research has started to shift toward the neurosciences using techniques such as neuroimaging and epigenetic analysis for the prediction and evaluation of therapy effects [22-24]. Whereas it is now widely accepted that psychotherapy has significant effects on brain function, the improvement of psychotherapeutic interventions based on neuroscientific findings is still in an early stage, and it appears important to provide a more systematic framework.

\section{Further Integration of Neurobiology and Psychotherapy}

This section proposes a refined concept for a systematic integration of neurobiology and psychotherapy (Fig. 1). Specifically, we propose to (1) use the RDoC of mental functioning as an example for a neurobiological framework as reference, (2) to then identify psychotherapeutic techniques that are thought to match to and modify these domains, and (3) finally give examples of how to integrate these into systematic augmentation research and clinical translation (Fig. 2). In other words, we propose to extend the $\mathrm{RDoC}$ to psychotherapy.

Of note, we selected a neurobiology and not a psychotherapy perspective as reference to facilitate the identification of neurobiological augmentation strategies, the main objective of our work. As this reference, we use the $\mathrm{RDoC}$ system as the currently best-refined neurobiological framework of mental functioning. For psychotherapy, to our knowledge, a comparable framework of core interventions does not exist at present. This may be due to many reasons, including ideological differences between schools and school-dependent differences in terminology, which may obscure communality.

\section{Methods}

Seven searches were conducted with the aim of identifying experimental studies investigating the effect of neurobiologically augmented psychotherapy, compared to nonaugmented psychotherapy, in 7 domains of functioning, namely, threat/anxiety, reward learning, declarative memory, cognitive control, social communication, sleep, and sensorimotor systems. The searches were conducted in October 2020 using the search engine PubMed. All 


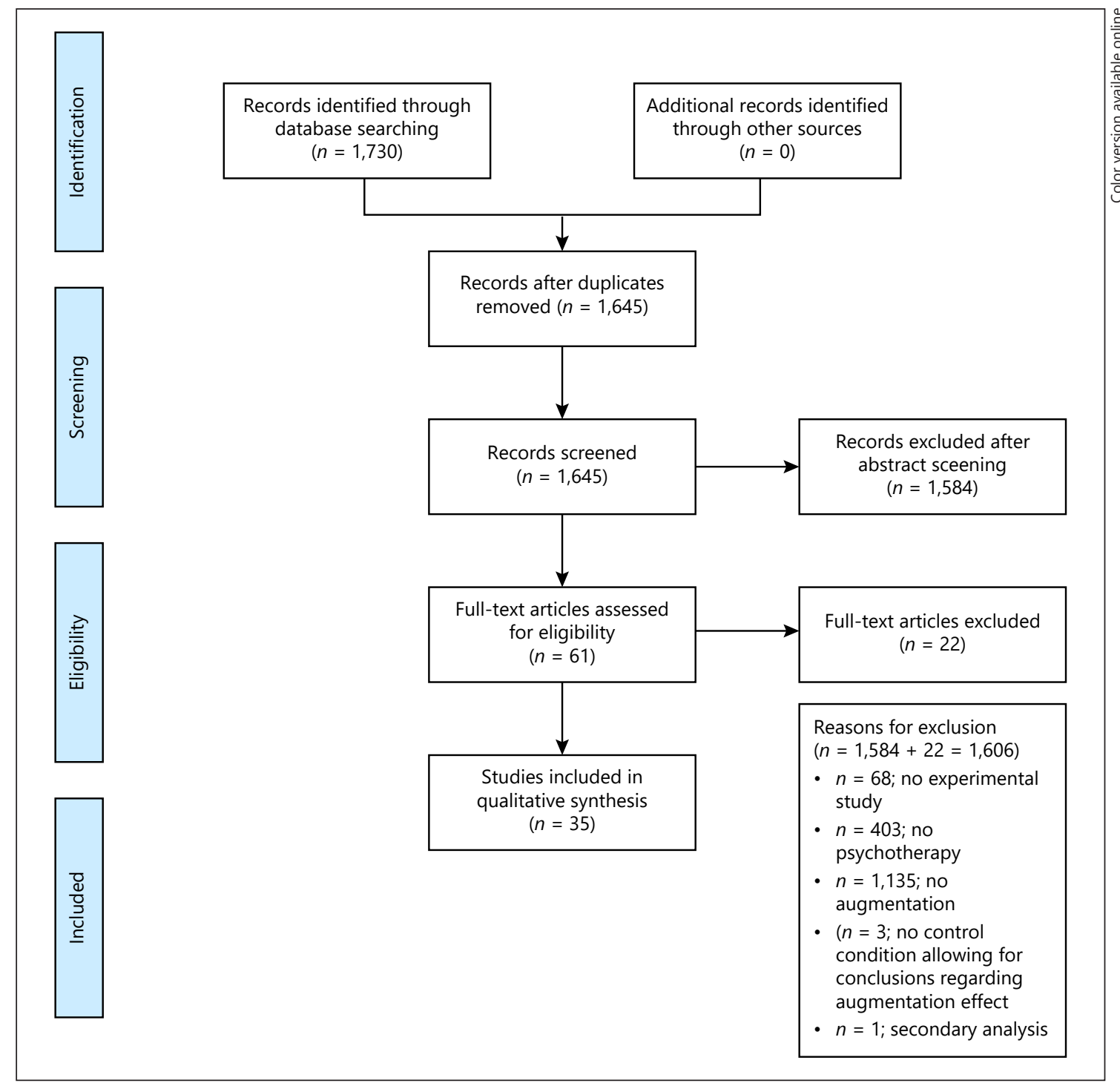

Fig. 1. Study flow.

searches were limited to controlled experiments. No restrictions regarding the date of publication were applied.

The following terms were searched for separately:

- ("threat" OR "anxiety" OR "fear") AND "psychotherapy" AND ("neuro*” OR "brain")

- "reward learning” AND "psychotherapy” AND (“neuro*” OR "brain")

- "declarative memory" AND "psychotherapy" AND ("neuro*” OR "brain")

- "cognitive control" AND "psychotherapy" AND ("neuro*” OR "brain")

- "social communication" AND "psychotherapy" AND ("neuro*” OR "brain")

- “sleep” AND “psychotherapy” AND (“neuro*” OR “brain”)
- "sensorimotor system" AND "psychotherapy" AND ("neuro*” OR "brain”)

Five authors (E.H., E.T., C.L.S., M.W., and K.F.) screened the titles and, where applicable, the abstracts and full texts of potentially eligible studies. Relevant data for the summary table (Table 1) were extracted from the full texts of selected studies. Doubts were discussed together with the first author $(\mathrm{EH})$ and last author $(\mathrm{CN})$ and resolved through decision by consensus.

The following inclusion criteria for primary studies were applied:

- primary study (no review, meta-analysis, comment etc.),

- experimental study with at least 2 groups (augmented and nonaugmented psychotherapy),

- investigation of the effect of a psychotherapeutic intervention, 


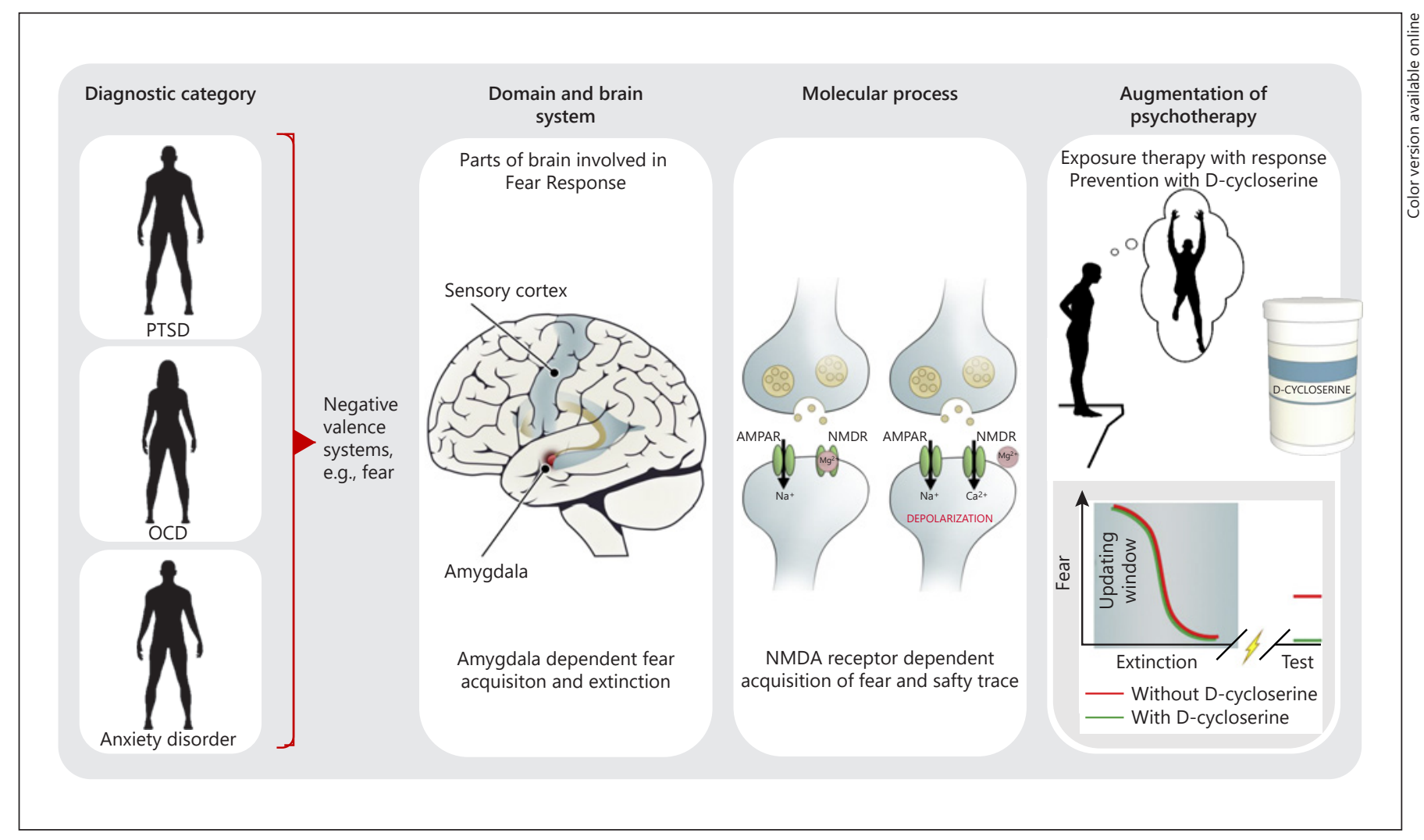

Fig. 2. Schematic illustration of neurobiology-informed psychotherapy. Figure annotations: The left side of the figure illustrates the standard categorical diagnostic approach of ICD-10 and the American DSM-5. Disadvantages of this approach are inhomogeneous categories/disorders and a poor fit with neurobiological concepts and mechanisms. The middle part of the figure illustrates the RDoC approach with basic domains of mental functioning and their underlying neurobiological mechanisms beyond diagnostic categories. The right part shows the idea of enhancing the efficacy of psychotherapy with the help of neuroactive compounds. Here, $\mathrm{d}$-cycloserine is shown as an example - other augmentation approaches are discussed in Table 1 and in the text. ICD, International Classification of Diseases; DSM, Diagnostic and Statistical Manual; RDoC, Research Domain Criteria; PTSD, posttraumatic stress disorder; OCD, obsessive-compulsive disorder.

\section{Results}

The flow of studies throughout search, title-screening, and full-text screening is detailed in Figure 1. The 7 searches combined resulted in 1,730 records, comprising 1,320 for threat/anxiety, 3 for reward learning, 10 for declarative memory, 48 for cognitive control, 10 for social communication, 399 for sleep, and 0 for sensorimotor systems. Thirty-five studies were included in the review and are detailed in Table 1; those were 29 on threat/anxiety, 3 on reward learning, 2 on cognitive control, and 1 on social communication. No studies were included for the remaining domains. In the following paragraphs, the main findings for each domain with included studies will be summarized (Fig. 2). 


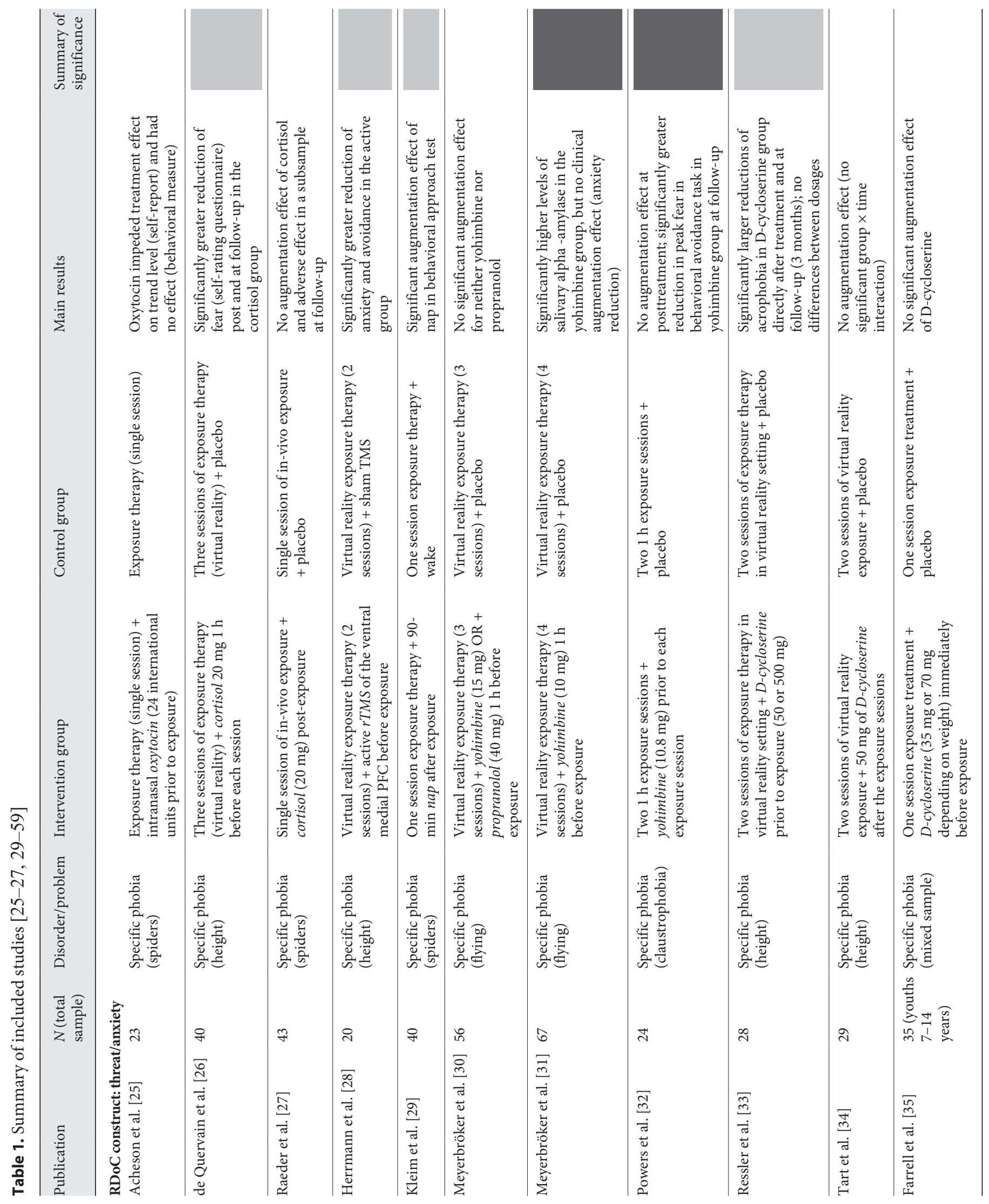




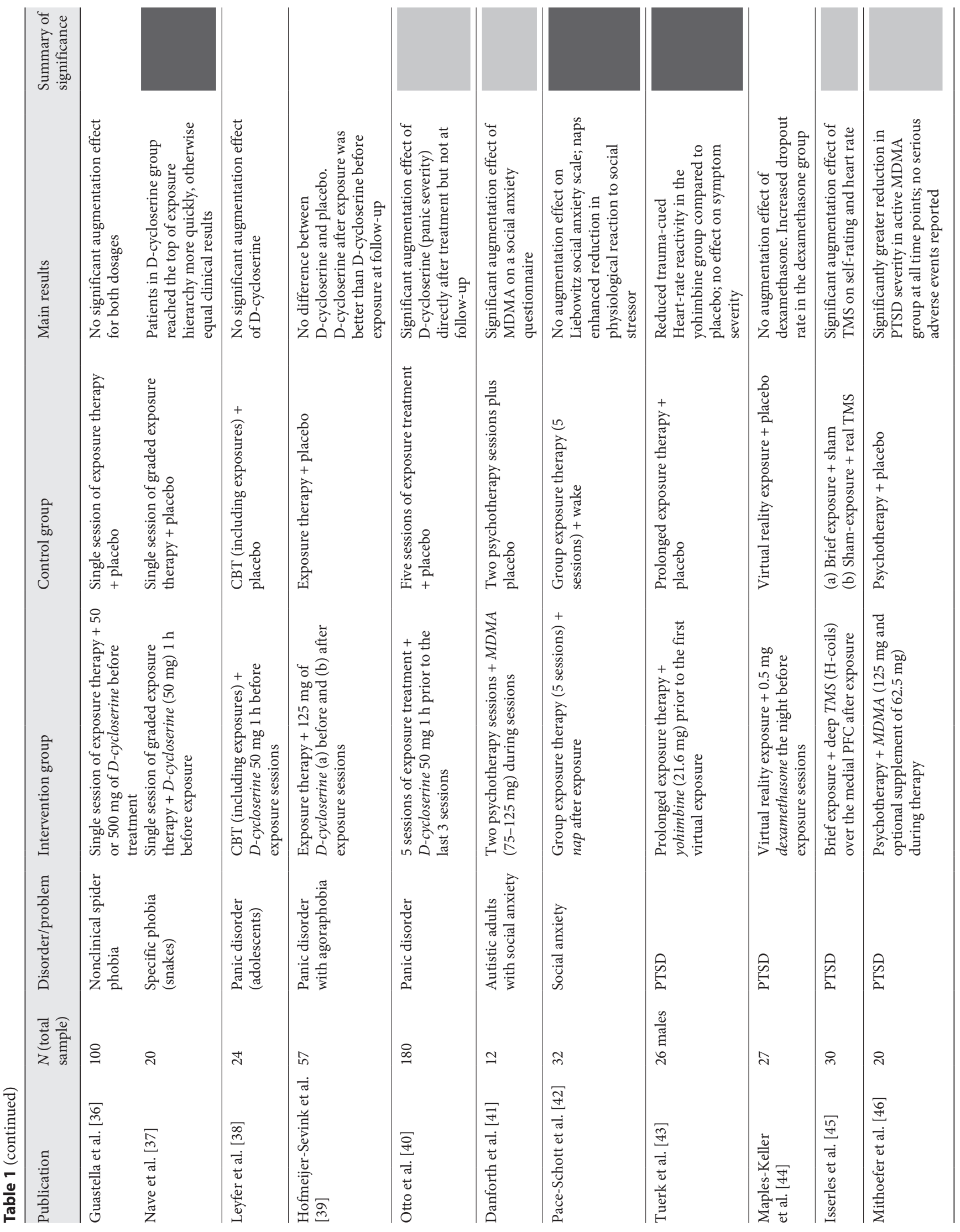




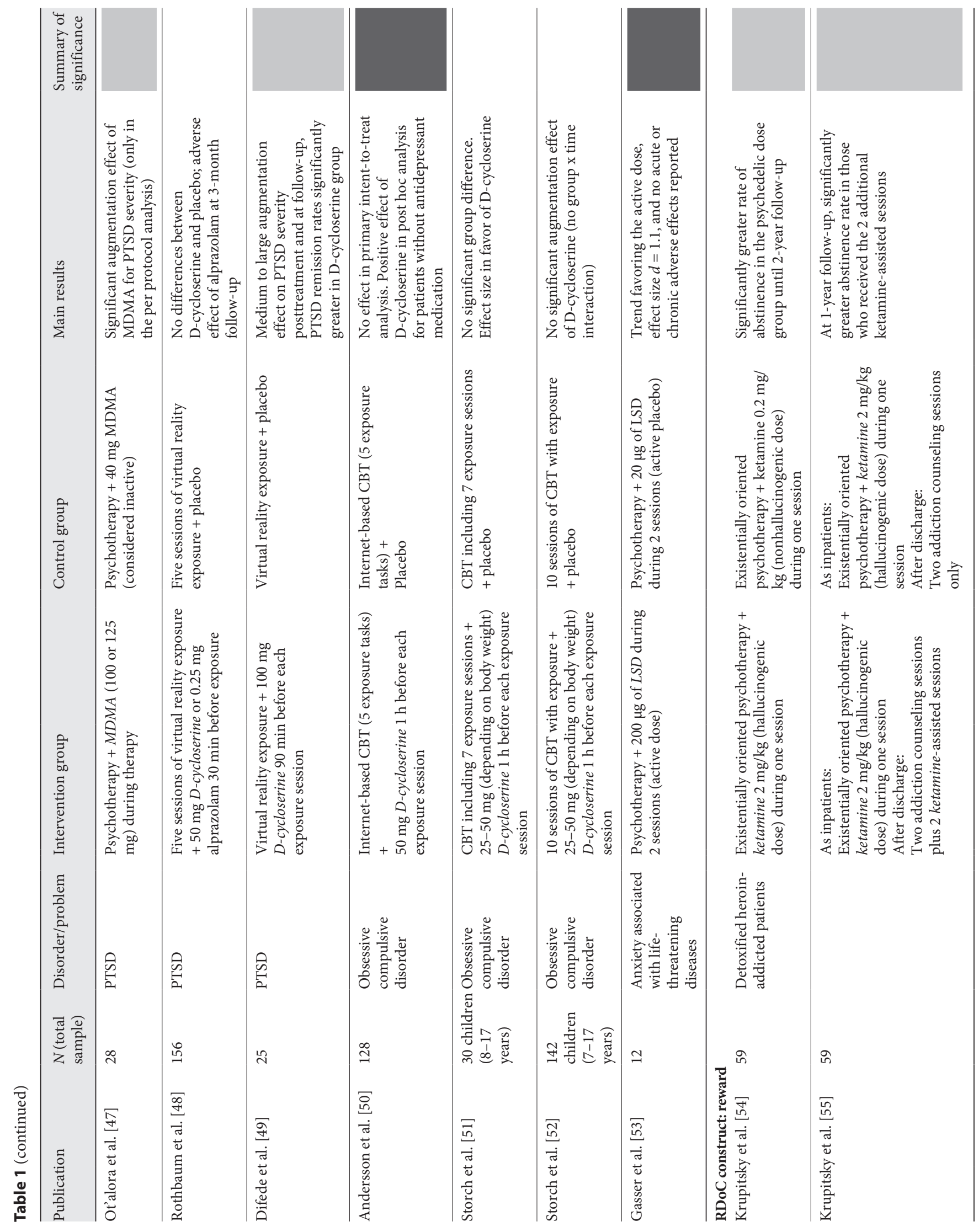




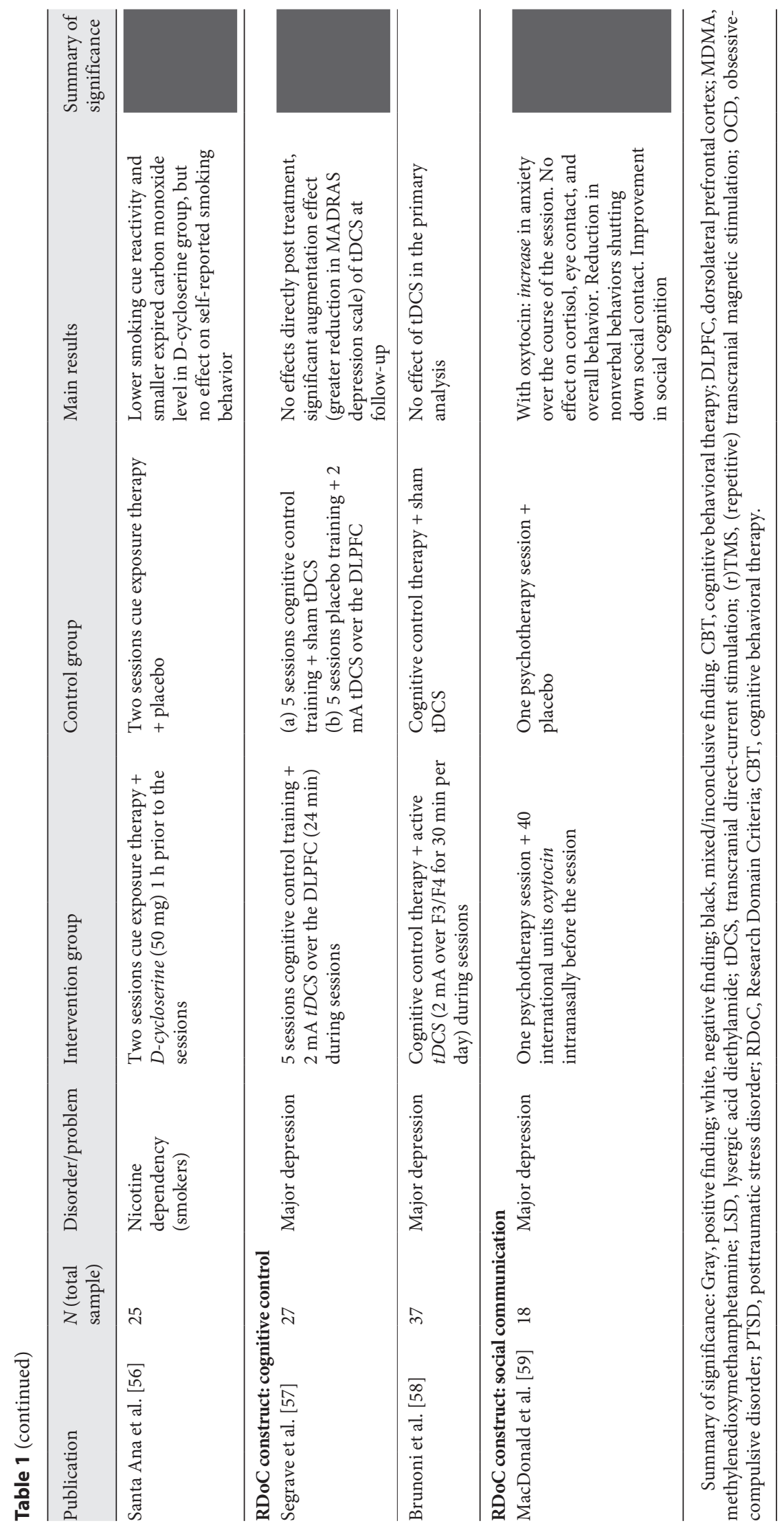




\section{Threat/Anxiety}

Two reactions to threat can be distinguished: fear, a normal reaction to an actual threat that is adaptive because it activates the body's defense system, and anxiety, a reaction to a potential, ambiguous, or uncertain threat that is often less adaptive and, when excessive, is associated with anxiety disorders. Anxiety disorders are characterized by excessive anxiety towards a specific stimulus, preoccupation with this stimulus, and efforts to avoid confrontation [60]. Exposure with response prevention is the gold standard psychotherapy for anxiety disorders [61]. Augmented exposure with response prevention has been investigated in patients with specific phobia (excessive fear of heights, spiders, snakes, etc., 13 studies), panic disorder (3 studies), social phobia (2 studies), posttraumatic stress disorder (PTSD, 7 studies), OCD (3 studies), and anxiety in the context of life-threatening somatic diseases (1 study). The probably best-characterized augmentation strategy is the administration of the NMDA receptor agonist D-cycloserine for fear extinction (13 studies). Assuming that maladaptive learning is the basis of excessive fear and anxiety, psychotherapy of anxiety can be understood as adaptive behavioral learning. The rationale of exposure therapy is to repeatedly confront the patient with fearevoking stimuli with the aim of gradually reducing fear, which is closely paralleled by fear extinction on a behavioral level [62]. From a neurobiological perspective, synaptic long-term potentiation (LTP), a persistent increase in synaptic strength, is a major molecular correlate of behavioral learning [63]. LTP in the lateral amygdala, which depends on the NMDA receptor, can be enhanced with the NMDA receptor agonist D-cycloserine [64]. In rats, systemic and intra-amygdala administration of D-cycloserine improves fear extinction [65]. Of note, fear extinction on the molecular level does not result from the extinction of the initial fear trace but from the consolidation of a novel safety memory trace. D-cycloserine alone does not have any anxiolytic effects, and continuous administration of D-cycloserine without psychotherapy is ineffective [66]. The efficacy of D-cycloserine augmentation in currently published studies is mixed - in specific phobia, panic disorder, and PTSD, part of the studies observed a significant augmentation effect, while others did not (Table 1). Efficacy does not seem to depend on dosage, since several studies tried different dosages from 50 to $500 \mathrm{mg}$ and found no dose-dependent effect. In OCD, all 3 included studies found no significant augmentation effect one, however, reported a trend toward significance [51] and another found a significant effect in a subgroup of patients without medication [50].
In other studies, other substances and methods were investigated with a comparable objective, namely, the augmentation of extinction learning in the process of exposure with response prevention. Those were the noradrenaline agonist yohimbine hydrochloride (3 studies in specific phobia, one in PTSD), the glucocorticoids cortisol and dexamethasone (2 studies in specific phobia and one in PTSD), and sleep (2 studies in specific phobia and social anxiety). Similar to D-cycloserine, the effects were mixed, with some significant and some insignificant studies.

In a minority of studies, other approaches beyond the augmentation of fear extinction learning have been investigated. One such approach is the augmentation of exposure therapy with repetitive transcranial magnetic stimulation. The rationale of this augmentation strategy is to increase the activity of the prefrontal cortex during or directly after exposure therapy. Transcranial magnetic stimulation augmentation was successful in one study in specific phobia [28] and another in PTSD [45]. A different approach is the administration of oxytocin during exposure therapy, with the aim of augmenting efficacy through an enhancement of neuroplasticity. This has been investigated in one study in specific phobia and was not successful [25]. Last, 4 studies have investigated methylenedioxymethamphetamine (MDMA) or the psychedelic drug lysergic acid diethylamide (LSD) as "catalysts" for psychotherapeutic experience, with the idea that increased social approach behavior and decreased feelings of anxiety may facilitate the psychotherapeutic process. The 4 studies in social anxiety disorder, PTSD, and anxiety associated with life-threatening diseases were all in favor of this approach (Table 1). In contrast to the other reviewed approaches, MDMA and LSD were not paired with exposure with response prevention, but with a supportive psychotherapy that aimed at assisting and reassuring patients in their experiential process after drug intake. Augmented sessions in these 4 studies took much longer than typical psychotherapy session (8-10 h plus an overnight stay).

\section{Reward Learning}

In contrast to threat/anxiety that belongs to the negative valence systems in the RDoC matrix, reward learning belongs to the positive valence systems. Reward learning describes a process by which humans learn to predict positive outcomes of their behaviors. It is a form of reinforcement learning through which behavior is modified. Reward learning is highly relevant for substance use disorders: through repeated intake of a specific substance such as alcohol or a drug, subjects learn that this intake leads 
to positive feelings and/or the absence of negative feelings. Combined with other features such as a quick onset of effect and tolerance, these rewarding effects account for the addiction potential of a substance.

Psychotherapy for patients with substance use disorders works mainly through motivational processes, stimulus control, and the reinforcement of alternative behaviors [67]. In 2 studies by the same work group, counseling for detoxified patients with heroin addiction resulted in a significantly higher rate of abstinence when combined with one ketamine-assisted session of existentially oriented psychotherapy. Potential mechanisms of action include an anti-craving effect of ketamine, its effects on neuroplasticity, or an effect of the psychedelic experience itself [55]. The exact mechanism of action is not yet known. A different augmentation approach investigated in one study is the augmentation of cue exposure (i.e., exposure to the craved substance without its intake) with D-cycloserine. Here, the assumed mechanism of action is similar to that described earlier for anxiety disorders, namely, an augmentation of extinction learning. One study of cue exposure for nicotine-dependency augmented by D-cycloserine brought mixed results (positive effect on physiology, but no effect on behavior) [56].

\section{Cognitive Control}

Cognitive control is a critical process necessary for goal-directed behavior. It is required when habitual strategies are not sufficient as a response to a novel or unclear situation, or when an appropriate response has to be selected from competing alternatives, for example, in the case of motivational conflicts. This function is impaired in patients with different mental disorders $[68,69]$. Promoting cognitive control and cognitive flexibility is a goal of psychotherapy, which is explicitly targeted, for example, in acceptance and commitment therapy with the help of metaphors and behavioral experiments [9]. It can be assumed that cognitive control plays a role in different mental disorders. Neuroimaging studies have demonstrated that cognitive control, at least in part, depends on activity changes in prefrontal cortical regions [70]. Interventional approaches that manipulate neural excitability, for example, with transcranial direct-current stimulation (tDCS), a noninvasive brain stimulation technique that uses a constant electrical current to modulate neuronal membrane potentials, might have the potential to improve cognitive control $[71,72]$. Augmented psychotherapy with the aim of improving cognitive control has been investigated in 2 studies in major depression. Both studies used tDCS over the prefrontal cortex during a cognitive training. Whereas Segrave et al. [57] found an augmentation effect, Brunoni et al. [58] found no effect with a similar protocol.

\section{Social Processes}

Social processes, that is, an individual's experience and behavior in interpersonal situations, are critically important for survival (e.g., infant-parent relationship), are impaired in many mental disorders, and influence vital other constructs such as motivation and reward (e.g., strive for social acceptance and recognition, and avoidance of rejection and threat). Thus, this $\mathrm{RDoC}$ construct is of high relevance for psychotherapy. Of note, unlike other RDoC constructs, social processes are not only a frequent content of psychotherapy (e.g., past and present significant interpersonal relationships) but directly influence the psychotherapeutic process itself since the quality of the alliance between the patient and therapist is an essential determinant of therapeutic success [73]. On a neurobiological level, animal studies have demonstrated that, for instance, the neuropeptide oxytocin, formed in the nucleus paraventricularis and nucleus supraopticus of the hypothalamus and released for example, during nursing and sexual behavior, promotes affiliation and attachment [74]. A proposed mechanism for this effect has been shown in a monogamous species of prairie voles: the activation of oxytocin receptors in the nucleus accumbens during interaction with a potential partner promotes activation of the dopaminergic reward system, leading to long-lasting attachment [75]. In a nonmonogamous (but otherwise largely similar) species of prairie voles, in contrast, the density of oxytocin receptors in the nucleus accumbens is reduced [76].

Modulating effects of oxytocin on social behavior have also been demonstrated in humans, such as enhanced expression of emotions [77], reduced attention toward angry in contrast to happy faces [78], increased response to social reward [79], and increase in prosocial behavior [80] after application of oxytocin. Since oxytocin can be applied intranasally in humans and has, at this point in time, no known major adverse effects, this opens up possibilities for a targeted use in the context of psychotherapy, for example, with the aim of increasing the patient's response to praise and reward or to increase the effects of social skills training. Interestingly, oxytocin could be applied in patients and therapists with the aim of improving the therapeutic relationship. We found one study investigating the effect of 40 international units of ocytocin, administered intranasally, for the augmentation of one session of psychotherapy in patients with depression [59]. The authors report mixed results such as a reduction in 
nonverbal behaviors aimed at shutting down social contact, an increase in anxiety over the course of the session, and no effect on overall behavior.

\section{Summary}

Neurobiologically augmented psychotherapy is best researched in the anxiety disorders. Here, the augmentation of exposure with response prevention by compounds and methods shown to enhance fear extinction learning is rather thoroughly investigated. Effects, however, are mixed: while some studies found positive results, an equal amount with comparable approaches and designs did not find any augmentation effects. Generally, augmentation effects for exposure therapy appear to be small. Future research needs to investigate predictors of response and nonresponse and potentially identify subgroups of patients with anxiety disorders for whom augmentation is effective. Another promising approach in the field is the enhancement of existentially oriented psychotherapy with psychedelics. Here, thorough monitoring of the patients during and after drug administration and potential side effects is of particular importance since psychedelic drugs have the potential for severe side effects especially when overdosed. It is to note that the mechanisms of action and the replicability of these studies are largely unknown. Promising, but not sufficiently researched, augmentation ideas are the enhancement of cognitive training with $\mathrm{tDCS}$ over the prefrontal cortex and the administration of oxytocin with the aim of facilitating social communication.

\section{Discussion}

This section discusses potential strengths and limitations that arise from the proposed allocation of psychotherapeutic processes to RDoC constructs. The systematic extension of the RDoC to psychotherapy discussed in this article is a novel way of integrating established psychotherapeutic techniques and new neurobiological interventions. Our work complements the currently most widely recognized matrix of neurobiological mechanisms of mental functioning with psychotherapy techniques and augmentation strategies. This approach is straightforward, provides an open matrix for further development, and has the direct potential to systematically guide future studies in healthy individuals and those with mental disorders. A particular strength is that treatment targets that are well characterized on various biological and behavioral levels can be modified on point with the help of defined psychotherapeutic techniques and aug- mentation strategies. Several aspects make the RDoC-informed psychotherapy particularly well suitable for the investigation of effective factors of psychotherapy, most importantly the direct and systematic integration of neurobiological and behavioral measures and the emphasis of single therapeutic techniques, instead of complex treatment packages. Modular psychotherapy is in line with recent advances in psychotherapy [81]. Here, aspects of functioning such as emotion regulation or social communication with high importance across different diagnoses are targeted with general psychotherapeutic interventions. This allows for more flexibility and individuality in the therapeutic process on the one hand and a more straightforward allocation to neurobiological mechanisms on the other hand.

RDoC-informed psychotherapy thus has the potential to guide future psychotherapy research to more comprehensive studies, investigating circumscribed, well-defined interventions and augmentation strategies, with known or assumed effects on one or more RDoC constructs, on various levels of analysis, in samples including healthy, subclinical, and impaired individuals. The targeted coadministration of compounds to psychotherapy, such as D-cycloserine, might reduce the necessity of long-term pharmacotherapy, such as with antidepressants or hypnotics, and associated side effects, such as sexual dysfunction or dependence, respectively.

Another strength is the potential for personalized medicine/psychotherapy. Some neural characteristics that (at this point in time) cannot be manipulated might serve to predict, select, and monitor treatment effects. For example, regarding the construct "declarative memory," patients with a certain brain-derived neurotrophic factor genetic polymorphism may be more likely to respond to an augmentation strategy (such as D-cycloserine), which aims at improving learning and memory. A future research agenda is outlined in Table 2 .

However, the approach raises many unresolved questions of clinical implementation. Many RDoC constructs are dimensional. A medium expression is often desired, whereas both extremely low and high expressions are typically classified as problematic. An example is fear (essential in case of real danger, but harmful if inappropriate or excessive). For clinical work with the RDoC, it may be necessary to establish measures of severity and cutoffs for the identification of individuals in need for treatment and to define strategies to prioritize domains in the context of complex mental health problems.

From a psychotherapeutic viewpoint, the RDoC constructs can be differentiated into those that are primarily 
Table 2. Proposed research agenda

Efficacy and side effects:

Proof of concept studies: investigate how analogues for defined pathological processes can be modulated in healthy humans. Examples are fear conditioning for "acute threat," the Paced Auditory Serial Addition Task (PASAT) for "frustrative nonreward," task switching (e.g., Wisconsin Card Sorting Test) for "cognitive control," and the interpretation of ambiguous communication situations for "social communication." Test the efficacy and side effects of different interventions, for example,: psychological interventions (exposure training, cognitive training, mindfulness training, social support, etc.), augmentation strategies (e.g., tDCS), and the combination of both against sham/placebo

Translation to clinical samples with impairment in one or more defined RDoC processes: use clinical outcomes such as symptom severity and quality of life. Investigate the efficacy and side effects of psychotherapy, alone and combined with augmentation strategies, against placebo

Personalized psychotherapy:

Identify risk factors for impairment (= pathological manifestation of one or more RDoC constructs): longitudinal population studies examining the predictive value of potential risk factors for the onset of pathology (e.g., gene methylation, brain volume and connectivity, and biographical and behavioral markers)

Identify predictors of response and nonresponse on different levels of analysis: analyze predictive value of neurobiological markers (e.g., BDNF and other genotypes, and volume and connectivity of relevant brain regions) for the outcome of augmented psychotherapy. Establish a framework for differential indication (which intervention for which patient)

Mechanisms:

Basic research examining how exactly brain stimulation and other augmentation strategies work

Dismantling studies aiming at the identification of key psychotherapeutic interventions that map to defined neural processes, for example, using interim measurement time points after every session and subjective ratings of patients and therapists regarding the efficacy of single interventions

Implementation:

(How) can RDoC be used for diagnosis and treatment indication in clinical practice?

Gather opinions of stakeholders, for example, patients, psychotherapists, nurses, and health insurance

Identify barriers regarding implementation and potential need for reconceptualization

Identify potential missing elements in the RDoC framework (e.g., shame, disgust, dissociation, pseudoneurological/conversion symptoms, and pain)

Conduct pilot projects evaluating the feasibility of RDoC-informed psychotherapy in clinical practice establish cutoffs and measures of severity for RDoC constructs

Select a target for augmentation: efficacy studies comparing the administration of, for example, oxytocin or tDCS to the patient, the therapist, or both

Examine interactions between psychotherapeutic interventions and augmentation strategies: pilot studies with close monitoring Examine interactions between different augmentation strategies: pilot studies with close monitoring, computer simulation, and animal studies

Examine interactions between different RDoC constructs: efficacy studies (see above) should use measures of different RDoC constructs as (secondary) outcomes

RDoC, Research Domain Criteria; tDCS, transcranial direct-current stimulation; BDNF, brain-derived neurotrophic factor.

suitable for patient-centered interventions and those that may be suitable for therapist-centered interventions. For instance, the domain of negative valence systems refers to processes that may occur in the patient (e.g., anxiety or loss). This domain appears to be suitable for patient-centered augmentation strategies. In contrast, the constructs of empathy and social communication relate to skills a therapist needs in order to conduct a successful therapy. These constructs may be suitable for therapist-related augmentation strategies. In a more complex view, there is no clear-cut limitation of domains to one participant of the interaction process in psychotherapy.

Neurobiology-Informed Psychotherapy
As a prerequisite for the implementation of RDoC-informed neuropsychotherapy, psychotherapeutic interventions that map to defined neural processes have to be further identified for targeted augmentation. However, this endeavor is challenging. Numerous classification schemes of psychotherapy have been suggested for training, clinical practice, and research - without ubiquitous acceptance today. Prominent examples include empirically supported therapies [82], the common factors [83], and modular psychotherapy $[81,84]$.

Other open questions are when to use which augmentation strategy and whether and how different strategies 
can be combined in one patient. For instance, D-cycloserine can be administered selectively after a successful psychotherapy session because it enhances the consolidation phase of newly induced LTP. However, defining a "successful" session is difficult because some interventions might induce transient worsening prior to improvement, and vice versa (e.g., in trauma therapy). A potential pitfall of the augmentation of fear extinction is that psychotherapy is a complex interpersonal process with limited predictability, which may sometimes result in unintended maladaptive learning experiences such as unmanageable fear and frustration in the context of an ERP session terminated ahead of schedule. Concerning augmentation, a potential solution to this problem is the selective administration of augmentation only after therapy sessions which are rated as successful [85]. On a neural level, after encoding, a memory trace is initially instable and requires a process of consolidation to become stable, resistant to interference, and accessible for later retrieval. D-cycloserine, as a NMDA receptor agonist, selectively promotes the consolidation phase of a newly encoded memory trace [86]. Thus, D-cycloserine can be administered after a successful psychotherapy session with the aim of promoting consolidation of adaptive new memory traces.

Overall, research into the effects of augmented psychotherapy is yet in an early stage. Exposure therapy augmented with D-cycloserine is to date the best-studied example. Here, significant effects compared to placebo have been demonstrated [4], but effects are small and potentially of limited clinical significance.

Several limitations need to be addressed. First, and importantly, the range of side effects is unclear and may include unexpected interactions between psychotherapy and augmentation strategies. Examples comprise the strengthening of unwanted memories (e.g., nap and trauma memory) and an overly strong bond with the therapist (e.g., oxytocin and problems of termination of the therapy). In addition, potential adverse carryover effects under circumstances of combined augmentation strategies, such as tDCS and D-cycloserine, are unclear. Moreover, $\mathrm{RDoC}$ constructs interact. This implies that modifying one construct will likely modify several other constructs. Future studies are needed to specify these interrelations. The RDoC framework that has been chosen as an example for a framework of neurobiological domains relevant for mental health is likely not covering all relevant aspects. The RDoC focuses on domains that can be described in healthy humans and may be on a quantitatively different, pathological level in patients, such as anxiety and sleep. However, some mental disorders, such as psychosis, are at least in part characterized by symptoms that are qualitatively different form healthy perception and behavior and thus cannot be described in healthy humans - such as delusions, hallucinations, and ipseity disturbance. Here, another classification framework such as the Systems Neuroscience of Psychosis (SyNoPsis) may be more appropriate [87].

Finally, ethical concerns arise from the possibilities of using the named augmentation strategies for neuroenhancement in healthy individuals. Potential areas of application would be cognitive enhancement, for example, in students and performance increase in athletes. We are committed to the concept of negative utilitarianism, that is, the notion that the use of the named interventions should be limited to the reduction of suffering, that is, the augmentation of therapy for patients with mental illness.

Together, RDoC-informed neuropsychotherapy might pave the way for more individualized ("precision") interventions and increased efficacy. However, to date, none of the approaches is evidence-based, approved, or reimbursed by health insurances. All applications are offlabel and should only run in a research context with careful monitoring and evaluation.

\section{Statement of Ethics}

The study is exempt from Ethical Committee approval (systematic review).

\section{Conflict of Interest Statement}

This research did not receive any specific grant from funding agencies in the public, commercial, or not-for-profit sectors. The authors have no conflicts of interest to report.

\section{Funding Sources}

No external funding has been received for this work.

\section{Author Contributions}

E.H., C.N., and D.R. have equally contributed to the conception of the work and the preparation of the manuscript. The other authors have been involved in the literature search, data extraction, integration of results, and preparation of the manuscript. 


\section{References}

1 Demyttenaere K, Bruffaerts R, Posada-Villa J, Gasquet I, Kovess V, Lepine JP, et al. Prevalence, severity, and unmet need for treatment of mental disorders in the World Health Organization World Mental Health Surveys. JAMA. 2004 Jun;291(21):2581-90.

2 Gelenberg AJ. A review of the current guidelines for depression treatment. J Clin Psychiatry. 2010 Jul;71(7):15.

3 Carpenter JK, Andrews LA, Witcraft SM, Powers MB, Smits JAJ, Hofmann SG. Cognitive behavioral therapy for anxiety and related disorders: a meta-analysis of randomized placebo-controlled trials. Depress Anxiety. 2018 Jun;35(6):502-14.

4 Mataix-Cols D, Fernández de la Cruz L, Monzani B, Rosenfield D, Andersson E, PérezVigil A, et al. D-Cycloserine augmentation of exposure-based cognitive behavior therapy for anxiety, obsessive-compulsive, and posttraumatic stress disorders: a systematic review and meta-analysis of individual participant data. JAMA Psychiatry. 2017 May 1; 74(5):501-10.

5 Kozak MJ, Cuthbert BN. The NIMH research domain criteria initiative: background, issues, and pragmatics. Psychophysiology. 2016 Mar;53(3):286-97.

6 Barlow DH. The oxford handbook of clinical psychology. Oxford University Press; 2014.

7 Winek JL. Systemic family therapy: from theory to practice. SAGE; 2009.

8 Raskin NJ, Rogers CR. Person-centered therapy. Current psychotherapies. 7th ed. Belmont, CA, USA: Thomson Brooks/Cole Publishing Co; 2005. p. 130-65.

9 Hayes SC. Acceptance and commitment therapy, relational frame theory, and the third wave of behavioral and cognitive therapies. Behav Ther. 2004;35(4):639-65.

10 Segal ZV, Williams M, Teasdale J. Mindfulness-based cognitive therapy for depression. 2nd ed. New York: Guildford Press; 2018. [cited 2020 Jul 1]. Available from: https:// www.guilford.com/books/MindfulnessBased-Cognitive-Therapy-for-Depression/ Segal-Williams-Teasdale/9781462537037.

11 Karpova NN, Pickenhagen A, Lindholm J, Tiraboschi E, Kulesskaya N, Agústsdóttir A, et al. Fear erasure in mice requires synergy between antidepressant drugs and extinction training. Science. 2011 Dec;334(6063):1731-

12 DeRubeis RJ, Siegle GJ, Hollon SD. Cognitive therapy versus medication for depression: treatment outcomes and neural mechanisms. Nat Rev Neurosci. 2008 Oct;9(10):788-96.

13 Linden DEJ. How psychotherapy changes the brain--the contribution of functional neuroimaging. Mol Psychiatry. 2006 Jun;11(6): 528-38.

14 Freud S. Die Traumdeutung. Gesammelte Werke II/III. London: Imago; 1900.

15 Watson JB. Psychology as the behaviorist views it. Psychol Rev. 1913;20(2):158.
16 Rohracher H. Die Arbeitsweise des Gehirns und die psychischen Vorgänge. Psyche Zeitschrift für Psychoanalyse. 1968;22(12): 978-9.

17 Birbaumer N. Furcht und Furchtlosigkeit: Zur Neurobiologie Des Bösen. Mainz: Akademie der Wissenschaften und der Literatur; 2002.

18 Kim S, Birbaumer N. Real-time functional MRI neurofeedback: a tool for psychiatry. Curr Opin Psychiatry. 2014 Sep;27(5):332-6.

19 Grawe K. Neuropsychotherapie. Göttingen: Hogrefe Verlag; 2004.

20 Etkin A, Pittenger C, Polan HJ, Kandel ER. Toward a neurobiology of psychotherapy: basic science and clinical applications. J Neuropsychiatry Clin Neurosci. 2005;17(2):145-58.

21 Kandel ER. A new intellectual framework for psychiatry. Am J Psychiatry. 1998 Apr;155(4): 457-69.

22 Dichter GS, Felder JN, Petty C, Bizzell J, Ernst M, Smoski MJ. The effects of psychotherapy on neural responses to rewards in major depression. Biol Psychiatry. 2009 Nov;66(9): 886-97.

23 McGrath CL, Kelley ME, Dunlop BW, Holtzheimer PE, Craighead WE, Mayberg HS. Pretreatment brain states identify likely nonresponse to standard treatments for depression. Biol Psychiatry. 2014 Oct;76(7): 527-35.

24 Ziegler C, Richter J, Mahr M, Gajewska A, Schiele MA, Gehrmann A, et al. MAOA gene hypomethylation in panic disorder-reversibility of an epigenetic risk pattern by psychotherapy. Transl Psychiatry. 2016 Apr;6:e773.

25 Acheson DT, Feifel D, Kamenski M, Mckinney R, Risbrough VB. Intranasal oxytocin administration prior to exposure therapy for arachnophobia impedes treatment response. Depress Anxiety. 2015 Jun;32(6):400-7.

26 de Quervain DJ, Bentz D, Michael T, Bolt OC, Wiederhold BK, Margraf J, et al. Glucocorticoids enhance extinction-based psychotherapy. Proc Natl Acad Sci U S A. 2011 Apr; 108(16):6621-5.

27 Raeder F, Merz CJ, Tegenthoff M, Wolf OT, Margraf J, Zlomuzica A. Post-exposure cortisol administration does not augment the success of exposure therapy: a randomized placebo-controlled study. Psychoneuroendocrinology. 2019 Jan;99:174-82.

28 Herrmann MJ, Simons BSE, Horst AK, Boehme S, Straube T, Polak T. Modulation of sustained fear by transcranial direct current stimulation (tDCS) of the right inferior frontal cortex (rIFC). Biol Psychol. 2018 Nov;139:173-7.

29 Kleim B, Wilhelm FH, Temp L, Margraf J, Wiederhold BK, Rasch B. Sleep enhances exposure therapy. Psychol Med. 2014 May; 44(7):1511-9.

30 Meyerbröker K, Morina N, Emmelkamp PMG. Enhancement of exposure therapy in participants with specific phobia: a randomized controlled trial comparing yohimbine, propranolol and placebo. J Anxiety Disord. 2018 Jun;57:48-56.

31 Meyerbroeker K, Powers MB, van Stegeren A, Emmelkamp PM. Does yohimbine hydrochloride facilitate fear extinction in virtual reality treatment of fear of flying? A randomized placebo-controlled trial. Psychother Psychosom. 2012;81(1):29-37.

32 Powers MB, Smits JA, Otto MW, Sanders C, Emmelkamp PM. Facilitation of fear extinction in phobic participants with a novel cognitive enhancer: a randomized placebo controlled trial of yohimbine augmentation. J Anxiety Disord. 2009 Apr;23(3):350-6.

33 Ressler KJ, Rothbaum BO, Tannenbaum L, Anderson P, Graap K, Zimand E, et al. Cognitive enhancers as adjuncts to psychotherapy: use of D-cycloserine in phobic individuals to facilitate extinction of fear. Arch Gen Psychiatry. 2004 Nov;61(11):1136-44.

34 Tart CD, Handelsman PR, Deboer LB, Rosenfield D, Pollack MH, Hofmann SG, et al. Augmentation of exposure therapy with post-session administration of D-cycloserine. J Psychiatr Res. 2013 Feb;47(2):168-74.

35 Farrell LJ, Waters AM, Oar EL, Tiralongo E, Garbharran V, Alston-Knox C, et al. D-cycloserine-augmented one-session treatment of specific phobias in children and adolescents. Brain Behav. 2018 Jun;8(6):e00984.

36 Guastella AJ, Dadds MR, Lovibond PF, Mitchell P, Richardson R. A randomized controlled trial of the effect of D-cycloserine on exposure therapy for spider fear. J Psychiatr Res. 2007 Sep;41(6):466-71.

37 Nave AM, Tolin DF, Stevens MC. Exposure therapy, D-cycloserine, and functional magnetic resonance imaging in patients with snake phobia: a randomized pilot study. J Clin Psychiatry. 2012 Sep;73(9):1179-86.

38 Leyfer O, Carpenter A, Pincus D. N-methyl$\mathrm{D}$-aspartate partial agonist enhanced intensive cognitive-behavioral therapy of panic disorder in adolescents. Child Psychiatry Hum Dev. 2019 Apr;50(2):268-77.

39 Hofmeijer-Sevink MK, Duits P, Rijkeboer MM, Hoogendoorn AW, van Megen HJ, Vulink NC, et al. No effects of D-cycloserine enhancement in exposure with response prevention therapy in panic disorder with agoraphobia: a double-blind, randomized controlled trial. J Clin Psychopharmacol. 2017 Oct;37(5):531-9.

40 Otto MW, Lee J, Hofmann SG, Hearon BA, Smits JA, Rosenfield D, et al. Examining the efficacy of d-cycloserine to augment therapeutic learning in depression. Contemp Clin Trials. 2016;48:146-52.

41 Danforth AL, Grob CS, Struble C, Feduccia AA, Walker N, Jerome L, et al. Reduction in social anxiety after MDMA-assisted psychotherapy with autistic adults: a randomized, double-blind, placebo-controlled pilot study. Psychopharmacology. 2018 Nov;235(11): 3137-48. 
42 Pace-Schott EF, Bottary RM, Kim SY, Rosencrans PL, Vijayakumar S, Orr SP, et al. Effects of post-exposure naps on exposure therapy for social anxiety. Psychiatry Res. 2018 Dec; 270:523-30.

43 Tuerk PW, Wangelin BC, Powers MB, Smits JAJ, Acierno R, Myers US, et al. Augmenting treatment efficiency in exposure therapy for PTSD: a randomized double-blind placebocontrolled trial of yohimbine $\mathrm{HCl}$. Cogn $\mathrm{Be}-$ hav Ther. 2018 Sep;47(5):351-71.

44 Maples-Keller JL, Jovanovic T, Dunlop BW, Rauch S, Yasinski C, Michopoulos V, et al. When translational neuroscience fails in the clinic: dexamethasone prior to virtual reality exposure therapy increases drop-out rates. J Anxiety Disord. 2019 Jan;61:89-97.

45 Isserles M, Shalev AY, Roth Y, Peri T, Kutz I, Zlotnick E, et al. Effectiveness of deep transcranial magnetic stimulation combined with a brief exposure procedure in post-traumatic stress disorder:-a pilot study. Brain Stimul. 2013 May;6(3):377-83.

46 Mithoefer MC, Wagner MT, Mithoefer AT, Jerome L, Doblin R. The safety and efficacy of $\{+/-\} 3,4-m e t h y l e n e d i o x y m e t h a m p h e t a m i n e-$ assisted psychotherapy in subjects with chronic, treatment-resistant posttraumatic stress disorder: the first randomized controlled pilot study. J Psychopharmacol. 2011 Apr;25(4):439-52.

47 Ot'alora GM, Grigsby J, Poulter B, Van Derveer JW, Giron SG, Jerome L, et al. 3,4-Methylenedioxymethamphetamine-assisted psychotherapy for treatment of chronic posttraumatic stress disorder: a randomized phase 2 controlled trial. J Psychopharmacol. 2018 Dec;32(12):1295-307.

48 Rothbaum BO, Price M, Jovanovic T, Norrholm SD, Gerardi M, Dunlop B, et al. A randomized, double-blind evaluation of D-cycloserine or alprazolam combined with virtual reality exposure therapy for posttraumatic stress disorder in Iraq and Afghanistan War veterans. Am J Psychiatry. 2014 Jun;171(6) 640-8.

49 Difede J, Cukor J, Wyka K, Olden M, Hoffman $\mathrm{H}$, Lee FS, et al. D-cycloserine augmentation of exposure therapy for post-traumatic stress disorder: a pilot randomized clinical trial. Neuropsychopharmacology. 2014 Apr; 39(5):1052-8.

50 Andersson E, Hedman E, Enander J, Radu Djurfeldt D, Ljótsson B, Cervenka S, et al. DCycloserine vs placebo as adjunct to cognitive behavioral therapy for obsessive-compulsive disorder and interaction with antidepressants: a randomized clinical trial. JAMA Psychiatry. 2015 Jul;72(7):659-67.

51 Storch EA, Murphy TK, Goodman WK, Geffken GR, Lewin AB, Henin A, et al. A preliminary study of D-cycloserine augmentation of cognitive-behavioral therapy in pediatric obsessive-compulsive disorder. Biol Psychiatry. 2010 Dec;68(11):1073-6.
52 Storch EA, Wilhelm S, Sprich S, Henin A, Micco J, Small BJ, et al. Efficacy of augmentation of cognitive behavior therapy with weight-adjusted d-cycloserine vs placebo in pediatric obsessive-compulsive disorder: a randomized clinical trial. JAMA Psychiatry. 2016 Aug; 73(8):779-88.

53 Gasser P, Holstein D, Michel Y, Doblin R, Yazar-Klosinski B, Passie T, et al. Safety and efficacy of lysergic acid diethylamide-assisted psychotherapy for anxiety associated with life-threatening diseases. J Nerv Ment Dis. 2014 Jul;202(7):513-20.

54 Krupitsky E, Burakov A, Romanova T, Dunaevsky I, Strassman R, Grinenko A. Ketamine psychotherapy for heroin addiction: immediate effects and two-year follow-up. J Subst Abuse Treat. 2002 Dec;23(4):27383.

55 Krupitsky EM, Burakov AM, Dunaevsky IV, Romanova TN, Slavina TY, Grinenko AY. Single versus repeated sessions of ketamineassisted psychotherapy for people with heroin dependence. J Psychoactive Drugs. 2007 Mar; 39(1):13-9.

56 Santa Ana EJ, Rounsaville BJ, Frankforter TL, Nich C, Babuscio T, Poling J, et al. D-Cycloserine attenuates reactivity to smoking cues in nicotine dependent smokers: a pilot investigation. Drug Alcohol Depend. 2009 Oct; 104(3):220-7

57 Segrave RA, Arnold S, Hoy K, Fitzgerald PB. Concurrent cognitive control training augments the antidepressant efficacy of tDCS: a pilot study. Brain Stimul. 2014 Apr;7(2):32531.

58 Brunoni AR, Boggio PS, De Raedt R, Benseñor IM, Lotufo PA, Namur V, et al. Cognitive control therapy and transcranial direct current stimulation for depression: a randomized, double-blinded, controlled trial. J Affect Disord. 2014 Jun;162:43-9.

59 MacDonald K, MacDonald TM, Brüne M, Lamb K, Wilson MP, Golshan S, et al. Oxytocin and psychotherapy: a pilot study of its physiological, behavioral and subjective effects in males with depression. Psychoneuroendocrinology. 2013 Dec;38(12):2831-43.

60 American Psychiatric Association. Diagnostic and statistical manual of mental disorders $\left(\mathrm{DSM}-5^{\circ}\right)$. Washington, D.C.: American Psychiatric Pub; 2013.

61 Carl E, Stein AT, Levihn-Coon A, Pogue JR, Rothbaum B, Emmelkamp P, et al. Virtual reality exposure therapy for anxiety and related disorders: a meta-analysis of randomized controlled trials. J Anxiety Disord. 2019 Jan; 61:27-36.

62 Morrison FG, Ressler KJ. From the neurobiology of extinction to improved clinical treatments. Depress Anxiety. 2014 Apr;31(4):27990.

63 Kandel ER. The molecular biology of memory storage: a dialogue between genes and synapses. Science. 2001 Nov;294(5544):1030-8.
64 Richter-Levin G, Maroun M. Stress and amygdala suppression of metaplasticity in the medial prefrontal cortex. Cereb Cortex. 2010 Oct;20(10):2433-41.

65 Walker DL, Ressler KJ, Lu KT, Davis M. Facilitation of conditioned fear extinction by systemic administration or intra-amygdala infusions of D-cycloserine as assessed with fear-potentiated startle in rats. J Neurosci. 2002 Mar;22(6):2343-51.

66 Heresco-Levy U, Javitt DC, Gelfin Y, Gorelik E, Bar M, Blanaru M, et al. Controlled trial of D-cycloserine adjuvant therapy for treatment-resistant major depressive disorder. J Affect Disord. 2006 Jul;93(1-3):239-43.

67 Dutra L, Stathopoulou G, Basden SL, Leyro TM, Powers MB, Otto MW. A meta-analytic review of psychosocial interventions for substance use disorders. Am J Psychiatry. 2008 Feb;165(2):179-87.

68 Martínez-Arán A, Penadés R, Vieta E, Colom F, Reinares M, Benabarre A, et al. Executive function in patients with remitted bipolar disorder and schizophrenia and its relationship with functional outcome. Psychother Psychosom. 2002 Feb;71(1):39-46.

69 Steinglass JE, Walsh BT, Stern Y. Set shifting deficit in anorexia nervosa. J Int Neuropsychol Soc. 2006 May;12(3):431-5.

70 Jung RE, Haier RJ. The Parieto-Frontal Integration Theory (P-FIT) of intelligence: converging neuroimaging evidence. Behav Brain Sci. 2007 Apr;30(2):135-87.

71 Mayseless N, Shamay-Tsoory SG. Enhancing verbal creativity: modulating creativity by altering the balance between right and left inferior frontal gyrus with tDCS. Neuroscience. 2015 Apr;291:167-76.

72 Hertenstein E, Waibel E, Frase L, Riemann D, Feige B, Nitsche MA, et al. Modulation of creativity with transcranial direct current stimulation. Brain Stimul. 2019 Sep-Oct;12(5): 1213-21

73 Barnicot K, Katsakou C, Bhatti N, Savill M, Fearns N, Priebe S. Factors predicting the outcome of psychotherapy for borderline personality disorder: a systematic review. Clin Psychol Rev. 2012 Jul;32(5):400-12.

74 Insel TR, Young LJ. The neurobiology of attachment. Nat Rev Neurosci. 2001 Feb;2(2): 129-36.

75 Liu Y, Wang ZX. Nucleus accumbens oxytocin and dopamine interact to regulate pair bond formation in female prairie voles. Neuroscience. 2003;121(3):537-44.

76 Ophir AG, Gessel A, Zheng DJ, Phelps SM. Oxytocin receptor density is associated with male mating tactics and social monogamy. Horm Behav. 2012 Mar;61(3):445-53.

77 Tops M, van Peer JM, Korf J. Individual differences in emotional expressivity predict oxytocin responses to cortisol administration: relevance to breast cancer? Biol Psychol. 2007 May;75(2):119-23. 
78 Domes G, Normann C, Heinrichs M. The effect of oxytocin on attention to angry and happy faces in chronic depression. BMC Psychiatry. 2016 Apr;16:92.

79 Nawijn L, van Zuiden M, Koch SB, Frijling JL, Veltman DJ, Olff M. Intranasal oxytocin increases neural responses to social reward in post-traumatic stress disorder. Soc Cogn Affect Neurosci. 2017 01;12(2):212-23.

80 Marsh N, Scheele D, Gerhardt H, Strang S, Enax L, Weber B, et al. The neuropeptide oxytocin induces a social altruism bias. J Neurosci. 2015 Nov;35(47):15696-701.
81 Schmahl C, Bohus M. Bedeutung der Neurobiologie für die modulare Psychotherapie. Nervenarzt. 2013 Nov;84(11):1316-20.

82 Chambless DL, Ollendick TH. Empirically supported psychological interventions: controversies and evidence. Annu Rev Psychol. $2001 \mathrm{Feb} ; 52(1): 685-716$.

83 Ahn H-n., Wampold BE. Where oh where are the specific ingredients? A meta-analysis of component studies in counseling and psychotherapy. J Couns Psychol. 2001;48(3):251-7.

84 Ellard KK, Fairholme CP, Boisseau CL, Farchione TJ, Barlow DH. Unified protocol for the transdiagnostic treatment of emotional disorders: protocol development and initial outcome data. Cogn Behav Pract. 2010 Feb; 17(1):88-101
85 Rothbaum BO. Critical parameters for D-cycloserine enhancement of cognitivebehaviorial therapy for obsessive-compulsive disorder. Am J Psychiatry. 2008 Mar; 165(3):293-6.

86 Kalisch R, Holt B, Petrovic P, De Martino B, Klöppel S, Büchel C, et al. The NMDA agonist D-cycloserine facilitates fear memory consolidation in humans. Cereb Cortex. 2009 Jan; 19(1):187-96.

87 Strik W, Stegmayer K, Walther S, Dierks T. Systems neuroscience of psychosis: mapping schizophrenia symptoms onto brain systems. Neuropsychobiology. 2017;75(3): 100-16. 